\title{
Research of Javascript technology in the development of e-commerce application
}

\author{
Haiyan Li \\ School Of Logistics And Electrical Commerce, Henan University of Animal Husbandry and \\ Economy, Zhengzhou 450044, China
}

haiyan@163.com

Keywords: Javascript, JQuery, Ajax. Electronic commerce, interaction

\begin{abstract}
The development of e-commerce is profoundly changing the pattern of the Internet, but also has great influence on the technology of making web sites. Javascript technology application in the e-commerce development greatly improve the interactive ability of websites, from the website of the UI design to asynchronous data interaction is inseparable from the Javascript technology. The appearance of the JQuery plugin Javascript programming easier, make data asynchronous interaction can even from the XML technology. In exploring the depth of future Javascript technology will bring more profound to the design of the e-commerce site.
\end{abstract}

\section{Introduction}

The development of the Internet is changing the way people live, people can buy and sell goods through the Internet, in fund management, real time communication with others, also can be a host of other activities. Enterprise first found and amplify the Internet characteristics of breakthrough time space limitations, electronic commerce arises at the historic moment [1]. E-commerce sites is the online platform for electronic commerce activity service. The emergence of e-commerce has injected fresh blood to the Internet economy, many enterprises have realized the traditional e-commerce giant computer, the construction of e-commerce sites generally. E-commerce portal has more users than the traditional company, especially the taobao reached tens of millions of visits, such a huge traffic is a huge pressure for the server. And the application of Javascript technology disperses the direct pressure to the server to the client.

\section{Organization of the Text}

Section Headings. Javascript technology overview. Javascript origins in 1992, about a company called Nombas developed a called C relief (C - minus - minus, Cmm) embedded scripting language. The language later renamed ScriptEase. As Netscape Netscape Navigator, surfing the Internet has become part of people's life and popular, while the demand for the development of a client-side scripting language is becoming more and more strong [2]. Due to slower speed, has been found in some when data is submitted to the server, is often spend more time to fill out the form, finally submitted to the server when the return error information, so that greatly reduces the visitors interact with the site of interest. Then Netscape and Sun jointly developed a special running on client scripting language called Javascript, the name and we know Java is not too big relations, just borrow more popular Java at that particular time. JavaScript 1.1 in 1997 as a draft submitted to the European computer manufacturers association (ECMA), the birth of ECMA - 262 standard, the standard defines new scripting language called ECMAScript, this also is the basis of JavaScript technology.

The emergence of Javascript technology achieved great success, the majority of developers began to web development perspective projection to the client. This download to the client through the browser, trigger when there is a need for new technology, greatly reduce the burden of the server, making large web application development becomes more efficient. Every client browse the website 
has become a small server, they can respond to user operation, preprocessing, submit to the server information to users according to the needs of the user to get data from the server.

\section{Javascript technology in the application of the e-commerce development}

Javascript application in UI design. E-commerce sites of the UI (User Interface) User Interface design is an important part of the site. E-commerce backend implementation can use Php, J2EE, Asp.net, and other technology, but the user to see the parts of the website mainly UI [3]. Good UI design can help keep your visitors in the first time, to let them continue to browse the website, and then choose to finish goods trade. At present more popular is flat web UI design, e-commerce sites UI design mainly concentrated in the navigation menu. Folding menu, drop-down menus, the effect of sliding door, such as focal figure is the most commonly used effect.

Folding menu production mainly use Javascript to control the Dom (document element model) elements of Css (cascading style sheets (Css) attribute value to control a particular web page elements show hidden.

Javascript in site application form is submitted. Forms are a e-commerce sites page one of the most common web page elements. Label is form: $<$ form $>$ form element $</$ form $>$. Form containing the form elements of a text field, text areas, buttons, radio buttons, choose more button group, etc. 12. The text field is the most commonly used, it is often used for users to log in, order to fill in and submit, messages, opinions, feedback, etc. Form is an important method of website to get the data from the client, the legitimacy of the form data validation becomes especially important. Illegal data submitted to the database will increase the redundancy of database, and may be some hackers use attack sites. The legality of the validation data method is divided into client and server, the server principle is the server for the client to submit the data to the server and then according to the legal rule check; The client's principle is before the form data submitted to the server by Javascript script to check the legitimacy of the data. The biggest difference between these two approaches is the client in the client to the server did not check before making a request for data, avoiding waste of bandwidth caused by data submitted to the server and the server process the data of pressure, of course, to be on the safe side should be retained in one of two ways. Here we'll look at how to check the legitimacy of a user registration information.

Javascript code:

<script type="text/javascript">function document.getElementById("username").value; document.getElementById("password").value;

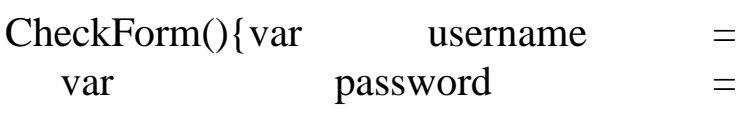

Code above is mainly for the user name, password, the length of a simple inspection, is thought by the Javascript document. The getElementByID ( ) method to get the value of the corresponding element in the form, then the length of the value judgment, only data is legal will be submitted to the server.

Javascript in the interaction of web application. As people constantly explore of Javascript technology, e-commerce website user experience also in continuous development, now people can easily like operating Windows desktop application on a web page to realize specific function. The goods add to cart, for example, by dragging to add and delete the items in the cart, popups dialog box, and so on. In theory we can use Javascript to achieve our in your web page in the Windows system is commonly used in operating habits, such as drag and drop, double-click, selected, etc. Before these functions in Javascript are unimaginable.

Javascript is realized in e-commerce sites is the most important interaction data interaction, the user evaluation and evaluation of the product page, according to product introduction and specification of asynchronous rendering these are used. We often say Ajax (short for Asynchronous JavaScript and XML) technology is the integrated use of JavaScript, XHTML and CSS and the DOM, 
XML, and XSTL XMLHttpRequest technology, etc. The technology has changed the traditional Web application using for asynchronous and synchronous interaction process program to request database. When you want to load the data. Not only fast, but also greatly reduces the burden on the server side. Such as the user to modify, and delete the items in the cart, do not need to refresh the entire page can complete [4].

Javascript code:

$<$ script

type="text/javascript">

\$(function()\{ \$("\#myselect").click(function()\{ \$.post("GetDatails.ashx",function(data,status)\{ if(sta tus=="success") $\{\$(" \#$ details").append("<tr $><\mathrm{td}>"+$ data+" $</ \mathrm{td}></$ tr $>$ "); $\}$ else $\{\quad$ alert("ajax false "); $\}\}) ;\}) ;\})$

$</$ script $>$

Javascript code writing and writing style is different, here is the JQuery framework written grammar. JQuery created by the americans, who in 2006, adhering to a core idea "write less, do more". JQuery is using Javascript technology developed by the class library, easier to write and to cross-browser compatible [5]. Written in the JQuery Ajax can greatly improve the efficiency, and even has a tendency to replace XML in Ajax. Only need to use a post method can transfer and facilitate access to data.

\section{Summary}

Javascript technology application in the e-commerce site, greatly improve the user experience, some based on the technology of flat design accepted by more and more users, followed by growth of site visits. In today's increasingly heated competition in the electricity, improve the user experience, a group of high loyalty is very important to the user. Of course, Javascript technology also has its own defects, the browser is set to disable scripting, so there was no way to run Javascript code; Javascript code to run on the client side, this virtually increases the burden of the client computer; Some malicious code to read the client information, violation of user privacy [6]. To solve all these problems also. Future Javascript technology development will gradually improve my own shortcomings, boost e-commerce sites to a more beautiful tomorrow.

\section{References}

[1] S.Y. Ceng: Journal of hubei college of finance and economics, 2012.2.25 (1).

[2] http://www.w3cschool.cn/js_intro.html [EB/OL]

[3] X. M. Wu, Y. G. Pan and X.F. Xie:Journal of wuhan university (science edition), 2012.10.

[4] H.C. Liao: Journal of electronic commerce, 2009 (10).

[5] H. P. Hou: Science wenhui, 2013.2.

[6] F. Peng, X.Y. Zhu: Journal of coal technology, 2012.5 (5). 